\title{
Dynamic study into autophagy and apoptosis during orthodontic tooth movement
}

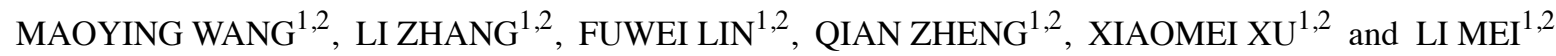 \\ ${ }^{1}$ Oral and Maxillofacial Reconstruction and Regeneration Laboratory, Southwest Medical University; ${ }^{2}$ Department of \\ Orthodontics, The Affiliated Stomatology Hospital of Southwest Medical University, Luzhou, Sichuan 646000, P.R. China
}

Received June 13, 2020; Accepted November 24, 2020

DOI: $10.3892 /$ etm.2021.9847

\begin{abstract}
Orthodontic tooth movement (OTM) has been widely observed worldwide. The OTM process is involved in several biological activities and can result in temporary hypoxia. The dynamic changes of autophagy and apoptosis during OTM have not, to the best of our knowledge, been previously reported. In the present study, an OTM animal model was established. Periodontal ligament cells (PDLCs) and osteoclasts were investigated using $\mathrm{H} \& \mathrm{E}$ and tartrate-resistant acid phosphatase staining. The changes in the expression levels of certain autophagy and apoptotic markers were investigated using immunohistochemical staining. A significant decrease in PDLC and an increase in osteoclast numbers were observed 1 day following OTM induction. The expression levels of Beclin-1 and LC3-II peaked at $1 \mathrm{~h}$ post-OTM, followed by a gradual decrease. The expression levels of P62 in each experimental group were significantly lower than those noted in the $0 \mathrm{~h}$ group. The expression levels of Bcl-2 were markedly increased $1 \mathrm{~h}$ following OTM and reached a maximum at 1 day post-OTM. The highest expression levels of Bax and caspase-3 were observed 7 days following OTM induction. The present study provided additional information regarding the involvement of autophagy and apoptotic markers in the OTM process and aided the understanding of the initiation and pathophysiological progression of this condition.
\end{abstract}

\section{Introduction}

Application of a controlled mechanical force can cause orthodontic tooth movement (OTM), which influences several biological processes. The biological changes to the periodontal ligament (PDL) during OTM can be divided into

Correspondence to: Dr Xiaomei $\mathrm{Xu}$, Oral and Maxillofacial Reconstruction and Regeneration Laboratory, Southwest Medical University, 2 Jiangyang South Road, Luzhou, Sichuan 646000, P.R. China

E-mail: xuxiaomei@hotmail.com

Key words: autophagy, apoptosis, reconstruction of periodontal ligament, orthodontic tooth movement the three following stages: Tissue degeneration, removal of necrotic tissue and new periodontal attachment $(1,2)$. OTM has been shown to be associated with the inflammatory response and cytokine production (3). The release of TGF- $\beta$ and IL-1 $\beta$ during the inflammatory response can facilitate osteoclast differentiation, bone remodeling and tooth movement $(4,5)$.

PDL can protect the alveolar bone against tooth root and is mainly composed of dense connective tissue (6). During orthodontic treatment, PDL is the initial biological medium that bears the mechanical force (7). Subsequently, the microenvironment of PDL cells (PDLCs) changes and the signal transduction system initiates the transformation of the mechanical stimulation signals into biochemical signals, participating in the reconstruction of periodontal tissues, such as PDL, alveolar bone and cementum $(8,9)$. During that process, cell death, bone formation, tissue absorption and regeneration promote OTM development (10). In addition, the PDL provides nutrition and maintains a balanced metabolism for periodontal cells and tissues, which in turn regulates periodontal remodeling (11).

Apoptosis and autophagy are the two main types of cell self-destruction (12). Apoptosis is a type I programmed cell death and is characterized by cell shrinkage, chromatin condensation, DNA degradation and fragmentation, cell division into apoptotic bodies and, ultimately, phagocytosis and degradation by phagocytes $(13,14)$. Autophagy is a type II programmed death and is a highly conserved intracellular evolutionary process (15). The autophagosome, which is comprised of a double-membraned structure, encapsulates longevity proteins and damaged organelles, and fuses with lysosomes (16). These findings indicate that apoptosis and autophagy may be closely associated with the process of OTM. However, the dynamic changes of autophagy and apoptosis with regards to the reconstruction of the PDL during OTM, to the best of our knowledge, have not been previously investigated.

The present study aims to investigate the dynamic changes in apoptosis and autophagy and the involvement of PDL and osteoclasts following OTM. The present study also aims to elucidate the mechanism of autophagy and apoptosis after OTM. Findings from the present study may provide potential targets for improving discomfort and reducing complications after orthodontic treatment. 


\section{Materials and methods}

Equipment and reagents. A NiTi spiral tension spring $(0.12 \mathrm{~mm})$ was purchased from Emondi Materials Technology Co., Ltd. The orthodontic ligation wire $(0.2 \mathrm{~mm})$ and dynamometer were purchased from Hangzhou Bioer Co., Ltd. The antibodies used in the present study were purchased from Abcam and were as follows: Beclin-1 (cat. no. ab232461; 1:1,500), LC3-II (cat. no. ab51520; 1:1,500), P62 (cat. no. ab56416; 1:1,000), Bcl-2 (cat. no. ab185002; 1:1,000), Bax (cat. no. ab32503; 1:1,500) and caspase-3 (cat. no. ab197202; 1:800). Tartrate-resistant acid phosphatase (TRAP) staining regents were obtained from Beijing Solarbio Technology Co., Ltd.

OTM animal model. In total, 40 male Sprague Dawley rats (6-8-week-old; weight, $247 \pm 33 \mathrm{~g}$ ) were purchased from the experimental animal center of Southwest Medical University and the animal study was approved by the Ethics Committee of Southwest Medical University (license no: SCXK-2019-17; Luzhou, China). The rats were housed under a 12-h light/dark cycle with free access to food and water at the temperature of $23-25^{\circ} \mathrm{C}$ and $40-50 \%$ humidity. The rats were randomly divided into 4 groups, with 10 rats in each group. Rats from the different groups were treated with orthodontic pressure for 0 or $1 \mathrm{~h}$, as well as 1 or 7 days. The animals were initially anesthetized by intraperitoneal injection with xylazine $(10 \mathrm{mg} / \mathrm{kg})$ and ketamine $(100 \mathrm{mg} / \mathrm{kg})$ as described previously (17). To check the successful induction of anesthesia, the palpebral reflex was tested by touching the medial canthus or the inner corner of the animals' eyes. No palpebral reflex indicated the successful induction of anesthesia. A nitinol tension spring was placed between the rat incisor and the right upper first molar. The two incisors were used as anchorage and a force of $0.392 \mathrm{~N}$ was applied to move the maxillary first molar (Fig. 1A). The force application device for each rat was monitored daily. The animals from the various groups were euthanized at the end of the testing time points ( $0,1 \mathrm{~h}, 1$ and 7 days). Inhalation of carbon dioxide (used at a $30 \%$ flow displacement rate) was used for animal euthanasia, which was performed in October 2019. Absence of respiration, heartbeat and the corneal/palpebral reflex indicated the death of animal. The right first molar and its surrounding tissues were dissected and subsequently used for other experiments. The relevant experiments involving the right first molar were subsequently performed (Fig. 1B).

Immunohistochemical (IHC) staining. The prepared tissues were fixed in $4 \%$ paraformaldehyde solution for $48 \mathrm{~h}$ at room temperature. The tissues were buffered in PBS solution for $24 \mathrm{~h}$ at room temperature and transferred into 14\% EDTA decalcification solution $(\mathrm{pH}:$ 7.3-7.5) for decalcification at room temperature. The decalcification solution was replaced daily. Following 6 weeks of decalcification, the tissues were embedded using paraffin wax and cut into $4-\mu$ m thick slices.

An initial deparaffinization step was conducted as follows: Slides were incubated in xylene twice (5 min each time), followed by washes in a descending ethanol gradient. The slides were then washed using deionized water twice $(5 \mathrm{~min}$ each time). In total, $3 \%$ hydrogen peroxide ( $25 \mathrm{~min}$ ) was used to remove endogenous peroxidase activity at room temperature.
Following washing with PBS (three times, $5 \mathrm{~min} /$ time), goat serum (cat. no. ab7481; Abcam) was applied for blocking (20 min) at room temperature. The aforementioned primary antibodies were incubated with the tissues overnight at $4^{\circ} \mathrm{C}$. Following washing with PBS (three times), the tissues were incubated with the horseradish peroxidase-conjugated secondary antibodies (cat. no. ab97051, 1:2,000; cat. no. ab205719, 1:2,000; Abcam) for $4 \mathrm{~h}$ at room temperature. Finally, DAB reagents were used, and slides were counterstained with hematoxylin for $20 \mathrm{sec}$ at room temperature. The relative protein expression was analyzed using Image Pro-Plus 6.0 (Media Cybernetics, Inc.) with a light microscope (magnifications, x200 and 400; BX51; Olympus Corporation). Briefly, the background of images was adjusted firstly. Five fields per slide were taken for further analysis. Regions of interest were selected and the intensity of DAB staining was calculated.

$H \& E$ staining. The tissues were prepared as aforementioned. Following deparaffinization, the tissues were stained with hematoxylin for $6 \mathrm{~min}$ at room temperature. After washing with running tap water for $5 \mathrm{~min}, 1 \%$ acid alcohol $(30 \mathrm{sec})$ was used for differentiation. After washing with running tap water again for $1 \mathrm{~min}, 0.2 \%$ ammonia water $(30 \mathrm{sec})$ was used for bluing. After washing with running tap water for $5 \mathrm{~min}$, slides were rinsed in $95 \%$ alcohol for five times. Finally, the tissues were stained with eosin for $20 \mathrm{sec}$ at room temperature, mounted and observed using light microscopy (magnifications, x200 and 400; BX51; Olympus Corporation). PDLCs were counted manually from five different fields of view per slide.

TRAP staining. The TRAP solution was made according to the instructions provided by the manufacturer. Following deparaffinization as aforementioned, the tissues were cultured with TRAP solution for $1 \mathrm{~h}$ at $37^{\circ} \mathrm{C}$ and washed with deionized water three times ( $5 \mathrm{~min} / \mathrm{each}$ time). Finally, the tissues were counterstained with hematoxylin for $3 \mathrm{~min}$ at room temperature, dehydrated and mounted. Tissues were observed using microscopy (magnifications, x200 and 400; BX51, Olympus Corporation). Osteoclasts were counted manually from five different fields of view per slide.

Measurement of tooth movement. The $\mathrm{mCT}$ system (Rigaku-mCT) was used to measure the tooth movement as previously described (18). After euthanizing at the end of testing time points $(0,1 \mathrm{~h}, 1$, and 7 days) before dissection, the mCT system was used to detect the distance between the mesial aspect of the second molar and the enamel on the most distal aspect of the first molar.

Western blot analysis. The tissue samples surrounding first molar were homogenized and then lysed using Protein Lysis Buffer (Promega Corporation). Protein concentration was measured using bicinchoninic acid protein assay method (Nanjing Jiancheng Bioengineering Institute). The same amount of protein $(30 \mu \mathrm{g})$ was separated using $10 \%$ SDS-PAGE. The proteins were transferred to a nitrocellulose membrane (EMD Millipore). Following blocking with 5\% non-fat milk prepared with TBS-Tween $20(0.1 \%)$ at room temperature for $2 \mathrm{~h}$, the membranes were incubated with the primary antibodies aforementioned overnight at $4^{\circ} \mathrm{C}$. After 
A

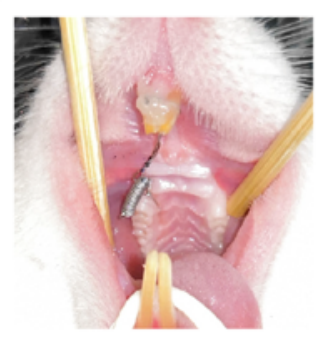

$\mathbf{B}$

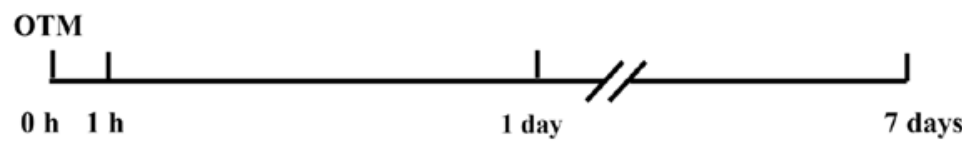

Animal sacrifice time points
C

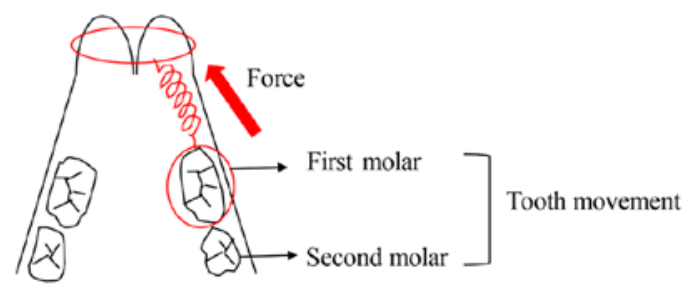

D

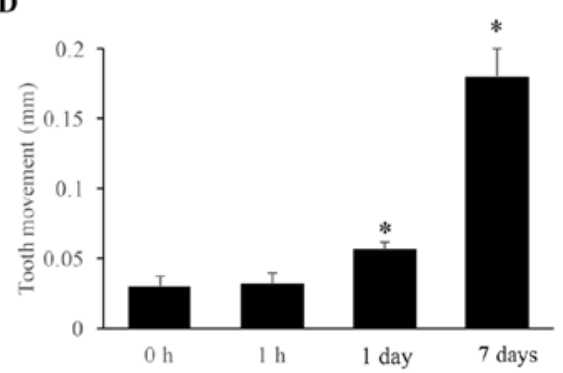

Figure 1. Establishment of the OTM animal model. (A) Representative image of the OTM animal model. (B) After 0, 1 h, 1 and 7 days post OTM, the number of PDLCs and osteoclasts and the levels of autophagy and apoptosis related proteins were measured. (C) Schematic illustration showing the measurement of tooth movement. Force was exerted in the direction of the red arrow to induce the movement of first molar. (D) Extent of tooth movement following OTM. ${ }^{*} \mathrm{P}<0.05$ vs. the $0 \mathrm{~h}$ group. OTM, orthodontic tooth movement; PDLCs, periodontal ligament cells.

washing, the membranes were incubated with the horseradish peroxidase-conjugated secondary antibodies (Goat anti-rabbit IgG; cat. no. ab97051; 1:2,000; Goat anti-mouse IgG, cat. no. ab205719, 1:2,000; Abcam) aforementioned for $2 \mathrm{~h}$ at room temperature. SuperSignal ${ }^{\mathrm{TM}}$ West Femto Maximum Sensitivity Substrate (Thermo Fisher Scientific, Inc.) was used as visualization reagent. ImageJ software 1.53 version (National Institutes of Health) was used to analyze the protein band intensity.

Reverse transcription-quantitative PCR (RT-qPCR). Total RNA was isolated using the TRIzol ${ }^{\circledR}$ reagent (Invitrogen; Thermo Fisher Scientific, Inc.) and the extracted RNA was reverse-transcribed into cDNA using SuperScript ${ }^{\mathrm{TM}}$ II Reverse Transcriptase kit (Invitrogen; Thermo Fisher Scientific, Inc.). The full temperature protocol for the reverse transcription was listed as follows: Primer annealing $\left(65^{\circ} \mathrm{C}, 5 \mathrm{~min}\right)$, DNA polymerization $\left(45^{\circ} \mathrm{C}, 50 \mathrm{~min}\right)$, enzyme deactivation $\left(85^{\circ} \mathrm{C}, 5 \mathrm{~min}\right)$. RT-qPCR was conducted using SYBR Premix Ex Taq ${ }^{\mathrm{TM}}$ II kit (Takara Bio, Inc.). The primer sequences used are as follows: GAPDH forward, 5'-ATG GGGAAGGTGAAGGTCG-3' and reverse, 5'-TCGGGG TCATTGATGGCAACAATA-3'; Bax forward, 5'-AGA CAGGGGCCTTTTTGCTAC-3' and reverse, 5'-AATTCG CCGGAGACACTCG-3'; Bcl-2 forward, 5'-GTGAACTGG GGGAGGATTGT-3' and reverse, 5'-GGAGAAATCAAA CAGAGGCCG-3'; caspase-3 forward, 5'-CTCGCTCTG GTACGGATGTG-3' and reverse, 5'-TCCCATAAATGA CCCCTTCATCA-3'; Beclin-1 forward, 5'-ATGGAGGGG TCTAAGGCGTC-3' and reverse, 5'-TGGGCTGTGGTA AGTAATGGA-3'; LC3-II forward, 5'-GACCGCTGTAAG GAGGTGC-3' and reverse, 5'-AGAAGCCGAAGGTTT CTTGGG-3'; and P62 forward, 5'-GAGGCACCCCGA
AACATGG-3' and reverse, 5'-ACTTATAGCGAGTTCCCA CCA-3'. GAPDH gene was used as internal control gene. The thermocycling conditions were set as follows: Initial denaturation $\left(98^{\circ} \mathrm{C}, 2 \mathrm{~min}\right) ; 35$ cycles for denaturation $\left(94^{\circ} \mathrm{C}\right.$, $30 \mathrm{sec})$, annealing $\left(55^{\circ} \mathrm{C}, 40 \mathrm{sec}\right)$ and extension $\left(72^{\circ} \mathrm{C}, 60 \mathrm{sec}\right)$; Final extension $\left(72^{\circ} \mathrm{C}, 5 \mathrm{~min}\right)$. The data were analyzed using the $2^{-\Delta \Delta \mathrm{Cq}}$ method (19).

Statistical analysis. The data were analyzed using SPSS 25.0 (IBM Corp.). One-way ANOVAs followed by post-hoc Tukey's test were used to analyze the significant differences between the various groups. $\mathrm{P}<0.05$ was considered to indicate a statistically significant difference.

\section{Results}

Establishment of the OTM animal model. To investigate the dynamic changes of autophagy and apoptosis during PDL reconstruction following OTM induction in rats, initially an OTM model was established (Fig. 1A). The levels of specific key autophagy and apoptotic proteins were measured at different time points post OTM (Fig. 1B). Moreover, the tooth movement was measured post-OTM induction and the data indicated that after 1 or 7 days of OTM, there was significant tooth movement compared with that in group $0 \mathrm{~h}$ (Fig. 1C and D).

Assessment of PDLC and osteoclast morphology using $H \& E$ and TRAP staining. The PDLCs were observed using $\mathrm{H} \& \mathrm{E}$ staining. PDLCs and PDL fibers in the control group were arranged in a regular fashion. With the extended treatment times, the PDL gap in the area where the pressure was exerted in the experimental groups was gradually narrowed and the 
A
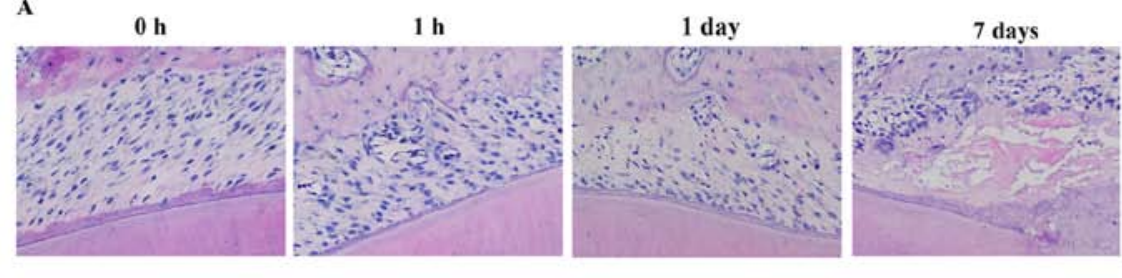

B

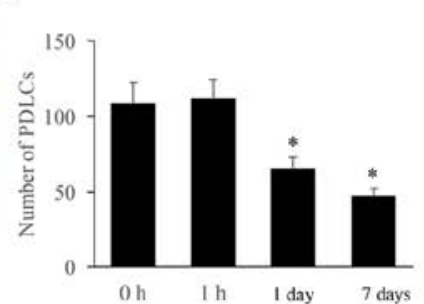

C

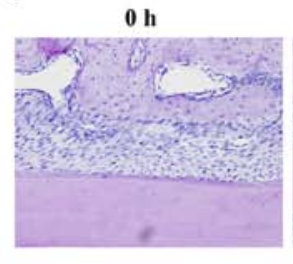
$1 \mathrm{~h}$

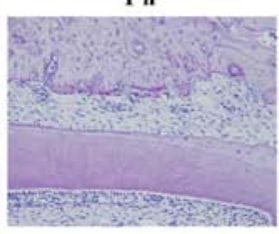

1 day

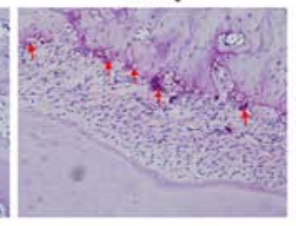

7 days

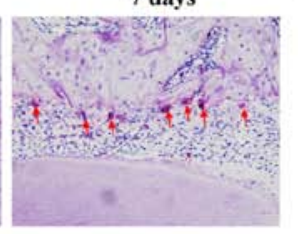

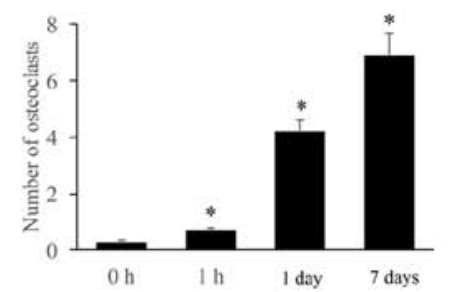

Figure 2. Morphological assessment of PDLCs and osteoclasts using H\&E and TRAP staining. (A) PDLCs were observed following H\&E staining. (B) Quantitative analysis of PDLCs. (C) Osteoclasts were observed following TRAP staining. (D) Quantitative analysis of osteoclasts. Red arrows indicate osteoclasts. ${ }^{*} \mathrm{P}<0.05$ vs. the $0 \mathrm{~h}$ group. PDLCs, periodontal ligament cells; TRAP, tartrate-resistant acid phosphatase.

local PDL fibers exhibited a wrinkled-like deformation. The PDLC arrangement was no longer regular, the blood vessels were compressed and the diameter of the tube was reduced (Fig. 2A). The number of PDLCs was slightly increased following $1 \mathrm{~h}$ of orthodontic force and significantly decreased following 1 and 7 days of treatment (Fig. 2B).

The morphology and quantity of osteoclasts were investigated using TRAP staining. The alveolar bone, in which osteoclasts were predominantly located, demonstrated apparent resorption lacuna (Fig. 2C). The number of osteoclasts in the pressure area was increased significantly following $1 \mathrm{~h}$ of orthodontic force application and reached its maximum value on day 7 (Fig. 2D).

Determination of expression levels of autophagy-related protein. Beclin-1, LC3-II and P62 were mainly expressed in the cytoplasm of PDLCs (Fig. 3A-C). The expression levels of Beclin-1 and LC3-II in the experimental groups reached their peak value following $1 \mathrm{~h}$ of treatment compared with that noted in the $0 \mathrm{~h}$ group (Fig. 3D). Subsequently, the expression levels of Beclin-1 and LC3-II decreased to the lowest levels on the 7th day (Fig. 3D). The changes in the expression levels of p62 in PDLCs were opposite to those noted for Beclin-1 and LC3-II. The expression levels of p62 in each experimental group were significantly lower than those noted in the $0 \mathrm{~h}$ group (Fig. 3D). At $1 \mathrm{~h}$ post-OTM treatment, the expression levels of P62 were at their lowest. Similar findings were observed by measuring the mRNA and protein expression levels of Beclin-1, LC3-II and p62. The expression levels of Beclin-1 and LC3-II were significantly increased following $1 \mathrm{~h}$ and 1 day of treatment (Fig. 3E and F). After 7 days, the levels of Beclin-1 and LC3-II reduced to baseline levels, possibly due to adaptation to continuous stress. However, the expression levels of p62 were inhibited following $1 \mathrm{~h}$ of treatment and were gradually increased following 1 day of treatment (Fig. 3E and F). After 7 days, the level of p62 returned to baseline levels, possibly due to adaptation to continuous stress.
Determination of the expression levels of apoptotic proteins. The expression levels of Bcl-2 significantly increased after induction of OTM $(1 \mathrm{~h})$ and reached a peak at the 1 day time-point of OTM treatment (Fig. 4A and D). Subsequently, the expression levels of $\mathrm{Bcl}-2$ were decreased following 7 days of OTM induction. However, a significant increase in the expression levels of Bax was observed only on the 7th day post-OTM (Fig. 4B and D). In addition, the expression levels of caspase- 3 fluctuated during the time course. The expression of caspase-3 was significantly increased at the 1-h and 7-day timepoints following OTM induction (Fig. 4C and D). A slight increase was noted following 1 day of OTM induction (Fig. 4C and D). The protein and mRNA expression levels of $\mathrm{Bcl}-2$, Bax and caspase-3 were also investigated using western blotting and RT-qPCR assays, respectively (Fig. 4E and F). Bcl-2 mRNA and protein levels, although raised, showed a reduction at 7 days, which was no longer significant for the protein levels compared with those at $0 \mathrm{~h}$. Caspase- 3 expression also stopped showing a significant raise at the 1 day compared with that at $0 \mathrm{~h}$, reducing from the $1 \mathrm{~h}$ time-point. However, this level was increased again at day 7. The mRNA levels of Bax were significantly decreased and increased following $1 \mathrm{~h}$ and 7 days of OTM induction, respectively (Fig. 4E and F). Meanwhile, the protein level of Bax was significantly increased after 7 days.

\section{Discussion}

Orthodontic mechanical force can be roughly divided into traction force and compression force, and it can affect the biological behavior of PDLCs $(20,21)$. Moreover, it has been reported that PDL hyalinization, bone density and bone turnover can affect the rate of OTM (22). Continuous orthodontic pressure can reduce the diameter of the blood vessels, lead to nutritional disorders, reduce metabolism of PDLCs and result in PDLC starvation (23-25). This nutrient starved environment can further induce autophagy (26). 
A

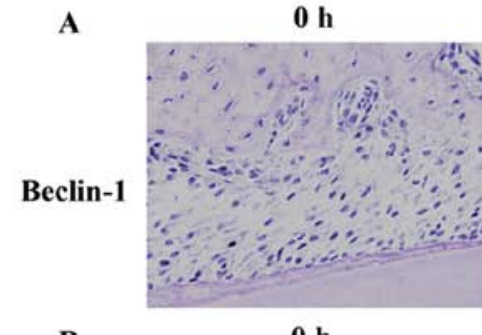

B

LC3 -II

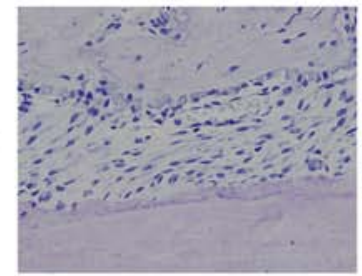

C

P62

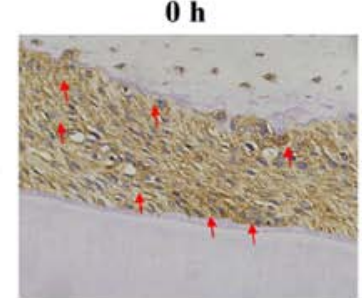

D

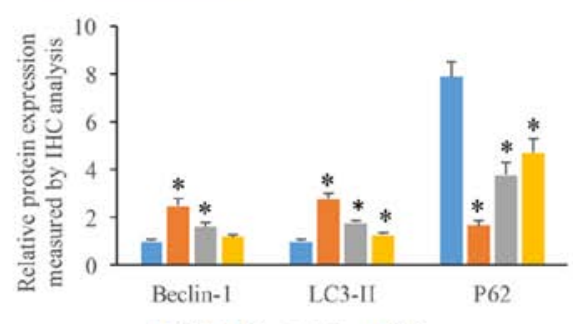

= $0 \mathrm{~h}=1 \mathrm{~h}=1$ day $=7$ days

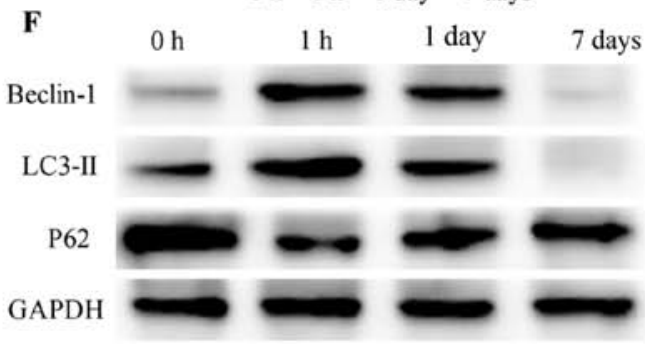

$1 \mathrm{~h}$

$1 \mathrm{~h}$

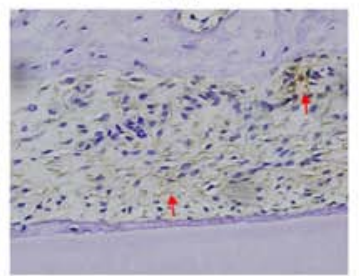

$1 \mathrm{~h}$
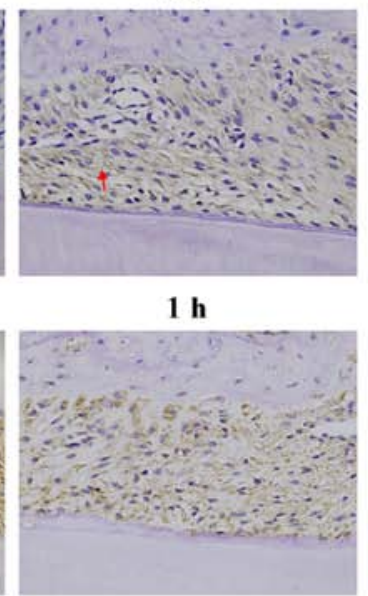

E
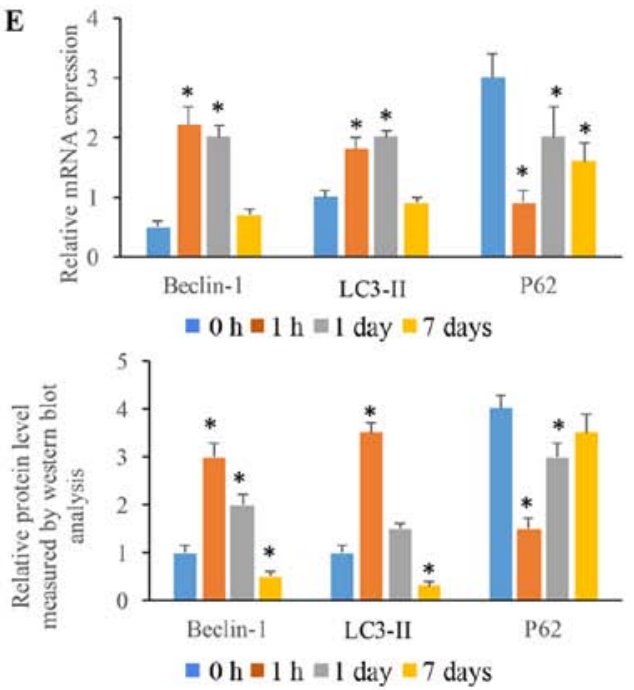

Figure 3. Measurement of the expression levels of autophagy proteins using IHC staining. (A) Beclin-1, (B) LC3-II and (C) P62 IHC staining. Red arrows indicate the strong positive cells measured by Beclin-1, LC3-II, or P62 IHC staining. (D) Quantitative analysis of Beclin-1, LC3-II and P62 staining. (E) The mRNA expression levels of Beclin-1, LC3-II and P62 were measured using reverse transcription-quantitative PCR. (F) The protein expression levels of Beclin-1, LC3-II and P62 were measured using western blot analysis. "P $<0.05$ vs. the $0 \mathrm{~h}$ group. IHC, immunohistochemical.

Autophagy is a physiological response of cells to stress and is maintained at a low baseline level under normal conditions. Several factors, such as mechanical stimulation and nutritional starvation can affect the induction of autophagy (23-25). It has been previously demonstrated that hypoxia and ischemia can result in an insufficient supply of energy to cells (27). Subsequently, autophagy is activated and the cell components are decomposed to provide energy, which is conducive to cell metabolism and survival (25). The induction of autophagy is involved in the regulation of the differentiation of osteoblasts (28) and osteoclasts (29), whereby the production and aggregation of osteoclasts is also associated with autophagy (30). Appropriate mechanical tension can promote the osteogenic differentiation of
PDL stem cells. In addition, autophagy can be activated by a compressive force on PDLCs (23). Autophagy regulates OTM by negatively modulating osteoclastogenesis and maintaining bone homeostasis (31). Sequestosome 1, a marker for autophagy, serves a key role during the stress adaptation of cells (32). Marked increases in sequestosome 1 levels in the PDL are observed after orthodontic force treatment (32). In addition, osteoclastogenesis can be promoted through the expression of autophagy-mediated RANKL after OTM (33). The active involvement of autophagy during OTM has been previously shown (31), but the dynamic changes to autophagy-related protein expression after OTM, to the best of our knowledge, have not been previously reported. 
A

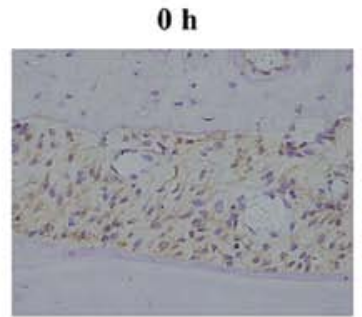

B

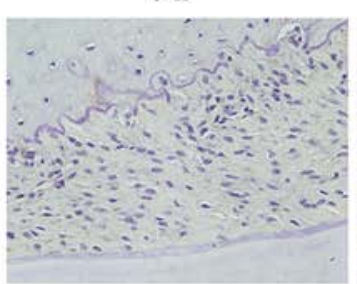

$\mathbf{0 ~ h}$

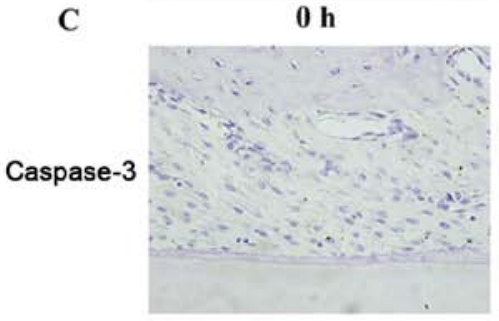

D
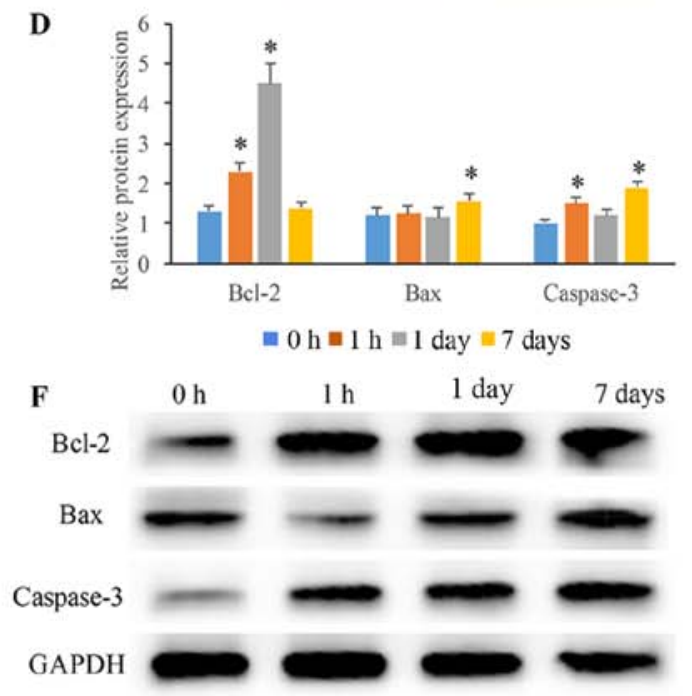

$1 \mathrm{~h}$

$1 \mathrm{~h}$

$1 \mathrm{~h}$
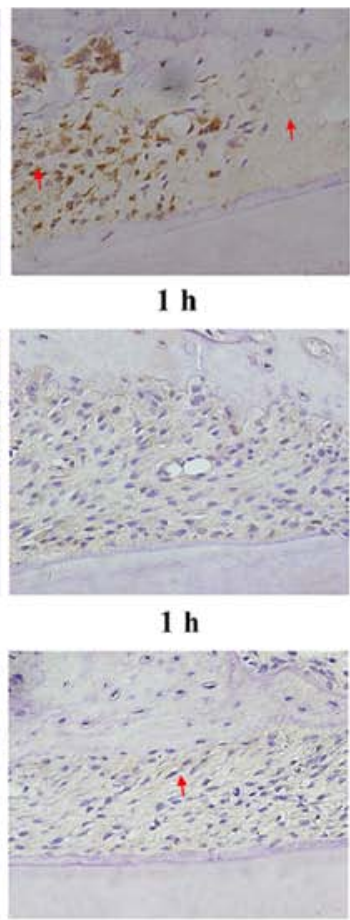

$\mathbf{E}$
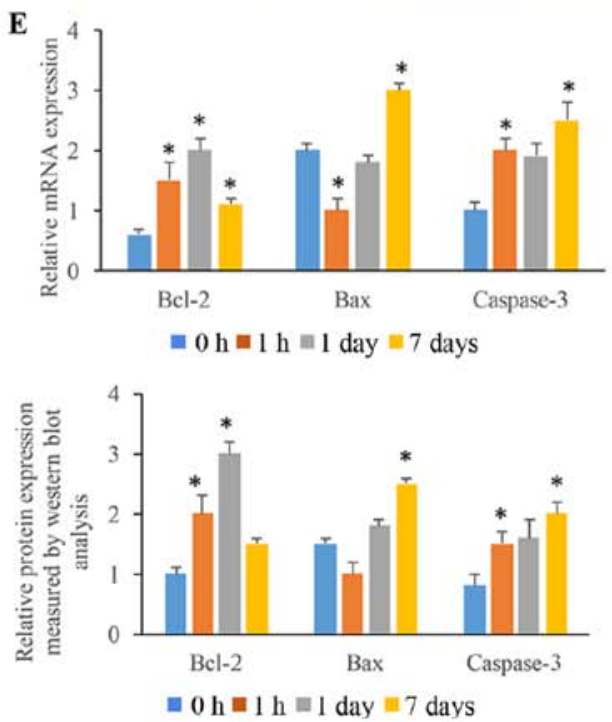

Figure 4. Induction of the expression of apoptotic proteins was measured using IHC staining. (A) Bcl-2, (B) Bax and (C) caspase-3 staining. Red arrows indicate the strong positive cells measured by Bcl-2, Bax, or Caspase-3 IHC staining. (D) Quantitative analysis of Bcl-2, Bax and caspase-3. (E) The mRNA expression levels of Bcl-2, Bax and caspase-3 were measured using reverse transcription-quantitative PCR. (F) The protein expression levels of Bcl-2, Bax and caspase- 3 were measured using western blot analysis. ${ }^{*} \mathrm{P}<0.05$ vs. the $0 \mathrm{~h}$ group. IHC, immunohistochemical.

The induction of apoptosis is characterized by chromatin condensation, nuclear fragmentation and decomposition of membrane-bound fragments $(34,35)$. Caspases cause the degradation of cellular proteins and activate specific endonucleases, which finally results in DNA fragmentation $(36,37)$. Hypoxic conditions comprise the main mechanism by which OTM can induce apoptosis and autophagy (38). It has reported that OTM may cause hypoxia of periodontal tissues and further trigger the release of hypoxia-inducible factor- $1 \alpha$, promoting osteogenic differentiation (1). A previous study indicated that Oridonin relieves hypoxia-induced apoptosis and autophagy by targeting the PI3K/AKT/mTOR signaling pathway and by promoting miR-214 expression (39). Therefore, induction of OTM may also influence the induction of apoptosis and autophagy through the regulation of the PI3K/AKT/mTOR signaling pathway and miR-214. In addition, hypoxia is believed to be an initiator for orthodontic tissue remodeling after OTM $(40,41)$. Hypoxia may accelerate the remodeling of the PDL and bone via inducing an aseptic inflammatory response (42). Therefore, the association between the findings of the present study and the aforementioned targets should be explored further.

Beclin-1 is a key regulator of autophagy and it is predominantly involved in the formation of the autophagosome (43). Downregulated or missing expression of Beclin-1 can result in abnormal autophagy function, which in turn induces 
apoptosis (44). Bcl-2 and Bax belong to the same family of proteins and the $\mathrm{Bcl}-2 / \mathrm{Bax}$ ratio is a key factor that determines the induction of apoptosis (45). Bcl-2 is not only an apoptosis suppressor gene, but also serves a regulatory role in the induction of autophagy by binding to Beclin-1 (46). Overexpression of Bcl-2 can inhibit the expression of Beclin-1 and suppress the formation of the autophagosome (47). In the present study, it was found that the levels of Bax and caspase- 3 were gradually increased, but the expression of $\mathrm{Bcl}-2$ was decreased to almost baseline levels 7 days after OTM. These data indicated that the induction of apoptosis by OTM was relatively slow compared to the induction of autophagy.

In the present study, continuous mechanical pressure appeared to induce autophagy during OTM. The expression levels of Beclin-1 and LC3-II were significantly increased following $1 \mathrm{~h}$ of OTM induction. However, following 7 days of OTM induction, the expression levels of Beclin-1 and LC3-II were markedly decreased, indicating the induction of PDLC apoptosis. Opposite expression changes of P62 compared to Beclin-1 and LC3-II were observed. The decrease of Beclin-1 and LC3-II, and the increase of P62 1 day after OTM may be due to the adaptive change of the cytoskeleton of PDLCs. However, the proteins levels of Beclin-1 and LC3-II became significantly reduced compared with those at baseline, where p62 expression was no longer significantly reduced at 7 days.

Long-term orthodontic stress stimulation and gradual aggravation of starvation can lead to the induction of PDLC autophagy and the expansion of the apoptotic response. Finally, hyaline degeneration of the PDL is observed during these processes $(48,49)$. A previous study suggested that LC3-II was markedly increased following hypoxia induction, which indicated that this process was significantly associated with the increased expression levels of autophagy-related proteins (50). In the present study, OTM treatment induced the expression of LC3-II following $1 \mathrm{~h}$ of OTM induction, indicating that it could cause the rapid stimulation of autophagy.

Caspase-3 is the key protease involved in apoptosis and it inhibits autophagy and promotes apoptosis by inactivating Beclin-1 (46,51). In addition, caspase-3 is the most important end shear enzyme involved in the process of apoptosis. It has been reported that activation of caspase- 3 precedes DNA fragmentation in apoptotic cells $(52,53)$. In the present study, the expression levels of caspase- 3 were significantly increased following $1 \mathrm{~h}$ of OTM induction and were gradually increased following the 1st day of the experimental model. The activation of caspase- 3 is indicative of the irreversible stage of apoptosis (54). In the present study, a significant increase in Bcl-2 expression levels was noted at the early stage post-OTM, which was accompanied by a gradual increase of both Bax and caspase-3.

In conclusion, the present study demonstrated a significant decrease in the number of PDLCs and a significant increase in the number of osteoclasts on the 1st day following OTM induction. Autophagy was rapidly initiated $1 \mathrm{~h}$ after OTM, whereas the induction of apoptosis was gradually increased after 7 days. The present study provided further evidence regarding the dynamic changes of autophagy and apoptosis during OTM in rats. In addition, there are some limitations to the present study. Firstly, only 4 time points were set in the animal experiments; therefore, some detailed changes to autophagy- and apoptosis-related proteins may have been overlooked. Secondly, further signaling pathways influencing the autophagy and apoptosis process after OTM need to be explored.

\section{Acknowledgements}

Not applicable.

\section{Funding}

The present study was supported by the scientific research fund project of Sichuan Medical Association (grant no. S18002) and applied basic research of Luzhou science and technology and Human Resources Bureau (grant no. 2018-JYJ-37).

\section{Availability of data and materials}

The datasets used and/or analyzed during the current study are available from the corresponding author on reasonable request.

\section{Authors' contributions}

MW and XX conceived and designed the experiments; MW, LZ, FL, LM and QZ performed the experiments, WM and XX wrote the paper. All authors have read and approved the final manuscript.

\section{Ethics approval and consent to participate}

The animal study was approved by the Ethics Committee of Southwest Medical University (license no: SCXK-2019-17; Luzhou, China).

\section{Patient consent for publication}

Not applicable.

\section{Competing interests}

The authors declare that they have no competing interests.

\section{References}

1. Zhang X, Chen D, Zheng J, Deng L, Chen Z, Ling J and Wu L: Effect of microRNA-21 on hypoxia-inducible factor-1 $\alpha$ in orthodontic tooth movement and human periodontal ligament cells under hypoxia. Exp Ther Med 17: 2830-2836, 2019.

2. de Jong T, Bakker AD, Everts V and Smit TH: The intricate anatomy of the periodontal ligament and its development: Lessons for periodontal regeneration. J Periodontal Res 52: 965-974, 2017.

3. Macari S, Ajay Sharma L, Wyatt A, Knowles P, Szawka RE, Garlet GP, Grattan DR, Dias GJ and Silva TA: Osteoprotective effects of estrogen in the maxillary bone depend on ER $\alpha$. J Dent Res 95: 689-696, 2016.

4. Castroflorio T, Gamerro EF, Caviglia GP and Deregibus A: Biochemical markers of bone metabolism during early orthodontic tooth movement with aligners. Angle Orthod 87: 74-81, 2017. 
5. Üretürk SE, Saraç M, Firatlı S, Can SB, Güven Y and Firatlı E: The effect of low-level laser therapy on tooth movement during canine distalization. Lasers Med Sci 32: 757-764, 2017.

6. Suttorp CM, Xie R, Lundvig DM, Kuijpers-Jagtman AM, Uijttenboogaart JT, Van Rheden R, Maltha JC and Wagener FA: Orthodontic forces induce the cytoprotective enzyme heme oxygenase-1 in rats. Front Physiol 7: 283, 2016.

7. Adnan S, Lone MM, Khan FR, Hussain SM and Nagi SE: Which is the most recommended medium for the storage and transport of avulsed teeth? A systematic review. Dent Traumatol 34: 59-70, 2018.

8. Yan XZ, van den Beucken J, Yuan C, Jansen JA and Yang F: Spheroid formation and stemness preservation of human periodontal ligament cells on chitosan films. Oral Dis 24: 1083-1092, 2018.

9. Onizuka S and Iwata T: Application of periodontal ligamentderived multipotent mesenchymal stromal cell sheets for periodontal regeneration. Int J Mol Sci 20: 2796, 2019.

10. Singh A, Gill G, Kaur H, Amhmed M and Jakhu H: Role of osteopontin in bone remodeling and orthodontic tooth movement: A review. Prog Orthod 19: 18, 2018.

11. Xu HY, Nie EM, Deng G, Lai LZ, Sun FY, Tian H, Fang FC Zou YG, Wu BL and Ou-Yang J: Periostin is essential for periodontal ligament remodeling during orthodontic treatment. Mol Med Rep 15: 1800-1806, 2017.

12. Ptak GE, Toschi P, Fidanza A, Czernik M, Zacchini F, Modlinski JA and Loi P: Autophagy and apoptosis: Parentof-origin genome-dependent mechanisms of cellular selfdestruction. Open Biol 4: 140027, 2014.

13. Gao L, Loveless J, Shay C and Teng Y: Targeting ROS-mediated crosstalk between autophagy and apoptosis in cancer. Adv Exp Med Biol 1260: 1-12, 2020

14. Li M, Tan J, Miao Y, Lei P and Zhang Q: The dual role of autophagy under hypoxia-involvement of interaction between autophagy and apoptosis. Apoptosis 20: 769-777, 2015.

15. Noguchi M, Hirata N, Tanaka T, Suizu F, Nakajima H and Chiorini JA: Autophagy as a modulator of cell death machinery. Cell Death Dis 11: 517, 2020

16. Wnuk A and Kajta M: Steroid and xenobiotic receptor signalling in apoptosis and autophagy of the nervous system. Int J Mol Sci 18: 2394, 2017.

17. Alexoff DL, Vaska P, Marsteller D, Gerasimov T, Li J, Logan J, Fowler JS, Taintor NB, Thanos PK and Volkow ND: Reproducibility of $11 \mathrm{C}$-raclopride binding in the rat brain measured with the microPET R4: Effects of scatter correction and tracer specific activity. J Nucl Med 44: 815-822, 2003.

18. Sahara N, Moriyama K, Iida M and Watanabe S: Fate of wornout functional teeth in the upper jaw dentition of sicyopterus japonicus (Gobioidei: Sicydiinae) during tooth replacement. Anat Rec (Hoboken) 301: 111-124, 2018.

19. Livak KJ and Schmittgen TD: Analysis of relative gene expression data using real-time quantitative PCR and the 2(-Delta Delta C(T)) method. Methods 25: 402-408, 2001.

20. Qin X, Li Q, Chen W, Bai Y, Baban B and Mao J: The circadian expression of osteogenic factors in periodontal tissue loading mechanical force: New concepts of the personalized orthodontic care. EPMA J 10: 13-20, 2019.

21. Li M, Yi J, Yang Y, Zheng W, Li Y and Zhao Z: Investigation of optimal orthodontic force at the cellular level through three-dimensionally cultured periodontal ligament cells. Eur J Orthod 38: 366-372, 2016.

22. Feng L, Yang R, Liu D, Wang X, Song Y, Cao H, He D, Gan Y, Kou X and Zhou Y: PDL progenitor-mediated PDL recovery contributes to orthodontic relapse. J Dent Res 95: 1049-1056, 2016.

23. King JS, Veltman DM and Insall RH: The induction of autophagy by mechanical stress. Autophagy 7: 1490-1499, 2011.

24. Ma KG, Shao ZW, Yang SH, Wang J, Wang BC, Xiong LM, Wu Q and Chen SF: Autophagy is activated in compression-induced cell degeneration and is mediated by reactive oxygen species in nucleus pulposus cells exposed to compression. Osteoarthritis Cartilage 21: 2030-2038, 2013.

25. Baskaran R, Poornima P, Priya LB, Huang CY and Padma VV: Neferine prevents autophagy induced by hypoxia through activation of Akt/mTOR pathway and Nrf2 in muscle cells. Biomed Pharmacother 83: 1407-1413, 2016.

26. Kuang Y, Hu B, Feng G, Xiang M, Deng Y, Tan M, Li J and Song J: Metformin prevents against oxidative stress-induced senescence in human periodontal ligament cells. Biogerontology 21: 13-27, 2020 .
27. Esumi H, Izuishi K, Kato K, Hashimoto K, Kurashima Y, Kishimoto A, Ogura $\mathrm{T}$ and Ozawa T: Hypoxia and nitric oxide treatment confer tolerance to glucose starvation in a 5'-AMP-activated protein kinase-dependent manner. J Biol Chem 277: 32791-32798, 2002.

28. Liu F, Fang F, Yuan H, Yang D, Chen Y, Williams L, Goldstein SA, Krebsbach PH and Guan JL: Suppression of autophagy by FIP200 deletion leads to osteopenia in mice through the inhibition of osteoblast terminal differentiation. J Bone Miner Res 28 2414-2430, 2013.

29. Xi G, Rosen CJ and Clemmons DR: IGF-I and IGFBP-2 stimulate AMPK activation and autophagy, which are required for osteoblast differentiation. Endocrinology 157: 268-281, 2016

30. Zhao Y, Chen G, Zhang W, Xu N, Zhu JY, Jia J, Sun ZJ, Wang YN and Zhao YF: Autophagy regulates hypoxia-induced osteoclastogenesis through the HIF-1 $/$ /BNIP3 signaling pathway. J Cell Physiol 227: 639-648, 2012.

31. Chen L, Mo S and Hua Y: Compressive force-induced autophagy in periodontal ligament cells downregulates osteoclastogenesis during tooth movement. J Periodontol 90: $1170-1181,2019$

32. Memmert S, Nogueira AVB, Damanaki A, Nokhbehsaim M, Rath-Deschner B, Götz W, Gölz L, Cirelli JA, Till A, Jäger A and Deschner J: Regulation of the autophagy-marker Sequestosome 1 in periodontal cells and tissues by biomechanical loading. J Orofac Orthop 81: 10-21, 2020.

33. Li W, Zhao J, Sun W, Wang H, Pan Y, Wang L and Zhang WB: Osteocytes promote osteoclastogenesis via autophagy-mediated RANKL secretion under mechanical compressive force. Arch Biochem Biophys 694: 108594, 2020.

34. Kijima M and Mizuta R: Histone H1 quantity determines the efficiencies of apoptotic DNA fragmentation and chromatin condensation. Biomed Res 40: 51-56, 2019.

35. Zierler S, Klein B, Furtner T, Bresgen N, Lütz-Meindl U and Kerschbaum HH: Ultraviolet irradiation-induced apoptosis does not trigger nuclear fragmentation but translocation of chromatin from nucleus into cytoplasm in the microglial cell-line, BV-2. Brain Res 1121: 12-21, 2006

36. Verma DK, Gupta S, Biswas J, Joshi N, Sivarama Raju K, Wahajuddin $M$ and Singh S: Metabolic enhancer piracetam attenuates the translocation of mitochondrion-specific proteins of caspase-independent pathway, poly [ADP-Ribose] polymerase 1 up-regulation and oxidative DNA fragmentation. Neurotox Res 34: 198-219, 2018.

37. Samejima K, Ogawa H, Ageichik AV, Peterson KL, Kaufmann SH, Kanemaki MT and Earnshaw WC: Auxin-induced rapid degradation of inhibitor of caspase-activated DNase (ICAD) induces apoptotic DNA fragmentation, caspase activation, and cell death: A cell suicide module. J Biol Chem 289: 31617-31623, 2014.

38. Luo G, Jian Z, Zhu Y, Zhu Y, Chen B, Ma R, Tang F and Xiao Y: Sirt1 promotes autophagy and inhibits apoptosis to protect cardiomyocytes from hypoxic stress. Int J Mol Med 43: 2033-2043, 2019

39. Gong L, Xu H, Zhang X, Zhang T, Shi J and Chang H: Oridonin relieves hypoxia-evoked apoptosis and autophagy via modulating microRNA-214 in H9c2 cells. Artif Cells Nanomed Biotechnol 47: 2585-2592, 2019.

40. Romer P, Wolf M, Fanghanel J, Reicheneder C and Proff P: Cellular response to orthodontically-induced short-term hypoxia in dental pulp cells. Cell Tissue Res 355: 173-180, 2014

41. Wolf M, Lossdörfer S, Römer P, Kirschneck C, Küpper K, Deschner J and Jäger A: Short-term heat pre-treatment modulates the release of HMGB1 and pro-inflammatory cytokines in hPDL cells following mechanical loading and affects monocyte behavior. Clin Oral Investig 20: 923-931, 2016.

42. Li Y, Jacox LA, Little SH and Ko CC: Orthodontic tooth movement: The biology and clinical implications. Kaohsiung J Med Sci 34: 207-214, 2018

43. Wang N, Zhang Q, Luo L, Ning B and Fang Y: $\beta$-asarone inhibited cell growth and promoted autophagy via $\mathrm{P} 53 / \mathrm{Bcl}-2 / \mathrm{Bclin}-1$ and P53/AMPK/mTOR pathways in human glioma U251 cells. J Cell Physiol 233: 2434-2443, 2018.

44. Abedin MJ, Wang D, McDonnell MA, Lehmann U and Kelekar A: Autophagy delays apoptotic death in breast cancer cells following DNA damage. Cell Death Differ 14: 500-510, 2007.

45. Huang Y, Fu Z, Dong W, Zhang Z, Mu J and Zhang J: Serum starvation-induces down-regulation of Bcl-2/Bax confers apoptosis in tongue coating-related cells in vitro. Mol Med Rep 17: 5057-5064, 2018 
46. Marino G, Niso-Santano M, Baehrecke EH and Kroemer G: Self-consumption: The interplay of autophagy and apoptosis. Nat Rev Mol Cell Biol 15: 81-94, 2014.

47. Zhu J, Cai Y, Xu K, Ren X, Sun J, Lu S, Chen J and Xu P: Beclin1 overexpression suppresses tumor cell proliferation and survival via an autophagy-dependent pathway in human synovial sarcoma cells. Oncol Rep 40: 1927-1936, 2018.

48. Xu J, Zhao X, Zeng J, Yu JH, Guan S, Xu XM and Mei L: Role of autophagy in the periodontal ligament reconstruction during orthodontic tooth movement in rats. J Dent Sci 15: 351-363, 2020

49. Memmert S, Damanaki A, Weykopf B, Rath-Deschner B Nokhbehsaim M, Götz W, Gölz L, Till A, Deschner J and Jäger A: Autophagy in periodontal ligament fibroblasts under biomechanical loading. Cell Tissue Res 378: 499-511, 2019.

50. Dong Y, Wu Y, Zhao GL, Ye ZY, Xing CG and Yang XD: Inhibition of autophagy by 3-MA promotes hypoxia-induced apoptosis in human colorectal cancer cells. Eur Rev Med Pharmacol Sci 23: 1047-1054, 2019.

51. Cao C, Wang W, Lu L, Wang L, Chen X, Guo R, Li S and Jiang J: Inactivation of Beclin-1-dependent autophagy promotes ursolic acid-induced apoptosis in hypertrophic scar fibroblasts. Exp Dermatol 27: 58-63, 2018.
52. Spiguel LR, Chandiwal A, Vosicky JE, Weichselbaum RR and Skelly CL: Concomitant proliferation and caspase-3 mediated apoptosis in response to low shear stress and balloon injury. J Surg Res 161: 146-155, 2010.

53. Shimizu H, Ohgoh M, Ikeda M, Nishizawa $Y$ and Ogura H: Caspase-3-like protease activity-independent apoptosis at the onset of neuronal cell death in the gerbil hippocampus after global ischemia. Biol Pharm Bull 30: 1950-1953, 2007.

54. Thrane C, Kaufmann U, Stummann BM and Olsson S: Activation of caspase-like activity and poly (ADP-ribose) polymerase degradation during sporulation in Aspergillus nidulans. Fungal Genet Biol 41: 361-368, 2004.

(i) $€$ This work is licensed under a Creative Commons Attribution-NonCommercial-NoDerivatives 4.0 International (CC BY-NC-ND 4.0) License. 